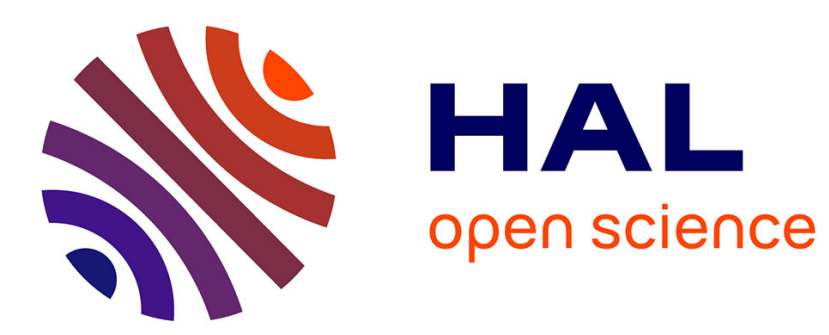

\title{
Gas heating in fast pulsed discharges in $\mathrm{N}-\mathrm{O}$ mixtures
}

\author{
A. Flitti, Sergey V. Pancheshnyi
}

\section{To cite this version:}

A. Flitti, Sergey V. Pancheshnyi. Gas heating in fast pulsed discharges in N-O mixtures. European Physical Journal: Applied Physics, 2009, 45 (2), pp.1. 10.1051/epjap/2009011 . hal-00480145

\section{HAL Id: hal-00480145 \\ https://hal.science/hal-00480145}

Submitted on 3 May 2010

HAL is a multi-disciplinary open access archive for the deposit and dissemination of scientific research documents, whether they are published or not. The documents may come from teaching and research institutions in France or abroad, or from public or private research centers.
L'archive ouverte pluridisciplinaire HAL, est destinée au dépôt et à la diffusion de documents scientifiques de niveau recherche, publiés ou non, émanant des établissements d'enseignement et de recherche français ou étrangers, des laboratoires publics ou privés. 


\title{
Gas heating in fast pulsed discharges in $\mathrm{N}_{2}-\mathrm{O}_{2}$ mixtures
}

\author{
Aicha Flitti ${ }^{\mathrm{a}}$ and Sergey Pancheshnyi ${ }^{\mathrm{b}}$ \\ LAPLACE, Université de Toulouse, CNRS-UPS-INP, 118 Route de Narbonne, 31062 Toulouse Cedex 9, France.
}

Received: December 15, 2008/ Revised version: 2

\begin{abstract}
The dynamics of gas heating in fast pulsed discharges in nitrogen-oxygen mixtures was analyzed using a state-specific plasmachemical model and an energy conservation equation. It was shown that the heating rate changes over time according to the changes of the species involved in the heating processes. An increasing dependence on time was shown. The results show that higher electron density, electric field or partial pressure of oxygen leads to a faster gas heating. However, the heating rate associated with excitation of vibrational and electronic degrees of freedom is almost independent of the value of the applied field in the studied range of fields.
\end{abstract}

PACS. 82.33.Xj Plasma reactions - 52.80.-s Electric discharges

\section{Introduction}

Gas heating in fast pulsed discharges can play an important role in certain applications including, for example, plasma-enhanced ignition of combustible mixtures, aerodynamic flow control, gas and surface treatment.

Electrons are usually the species which transmit the applied electrical energy to gas and branching between different mechanisms depends mainly on the electron en-

a Permanent address: LMSE, Faculté de Génie Électrique,

USTO-MB, BP 1505 El M'Naouar, 31000 Oran, Algeria.

b Corresponding author: sergey.pancheshnyi@laplace.univtlse.fr. ergy in the discharge. Three sub-systems are usually selected: (i) elastic collisions and rotational excitation, (ii) vibrational excitation and (iii) excitation of electronic levels including dissociation, ionization and attachment processes.

This division is caused by energy branching to corresponding degrees of freedom of gas which changes with a reduced electric field $E / N$, the ratio of the electric field $E$ and gas density $N$. The energy branching in different collisions of electrons with molecules in dry air $\left(\mathrm{N}_{2}-\mathrm{O}_{2}, 4: 1\right)$ at room temperature is presented in Figure 1. These results were obtained using BOLSIG + solver from the Boltzmann 


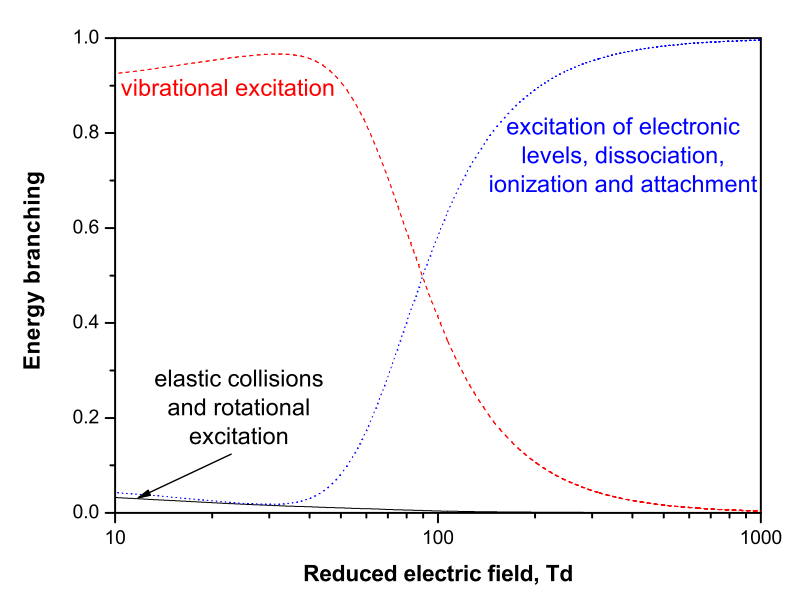

Fig. 1. Energy branching in electron collisions with molecules between (i) elastic collisions and rotational excitation, (ii) vibrational excitation and (iii) excitation of electronic levels including dissociation, ionization and attachment processes in non-excited dry air; $1 \mathrm{Td}=10^{-17} \mathrm{~V} \mathrm{~cm}^{2}$.

equation for electrons in weakly ionized gases [1]. The energy branching presented in Figure 1 was obtained neglecting super-elastic collisions, i.e. the collisions of electrons with excited heavy species in which the electrons gain energy. These collisions can significantly effect the energy branching under conditions of strong vibrational or electronic excitation of gas (see, for example, [2]) and will be considered in the present paper.

Elastic collisions of an electron with a heavy species cause a transfer of $2 m / M$ of incidental electron energy to translational degree of freedom of gas, where $m / M$ is the ratio of the electron mass to the mass of the heavy partner. Relaxation of translational excitation (TT) of the heavy species occurs in a few collisions between them (see, for example, [2]), so the energy transferred in elastic collisions can be directly associated with gas heating. Similarly, the electron energy spent on rotational excitation of molecules is redistributed in fast rotational-translational relaxation (RT) which takes place in a few dozen of molecular collisions [2]. In spite of the high speeds of these TT and RT processes (i), they are usually only significant for very low electric fields. The results presented in Figure 1 show that the energy loss by electrons in elastic collisions and in rotational excitation constitutes only a small percentage of total energy for a field of $10 \mathrm{Td}$ and decreases to almost negligible levels for higher fields.

At the same time, the amount of energy depositing into internal degrees of freedom of gas increases significantly with an increase in the electric field. Excitation of vibrations (ii) is the most energy-consuming process for molecular gases in low and elevated fields. The relaxation process of vibrational degree of freedom occurs in vibrationaltranslational (VT) collisions between molecules and other heavy species (molecules, atoms, etc.). The rate of VT relaxation depends heavily on the nature of the excited molecules and the composition of the plasma and can be quite low - usually, on a microsecond time scale at atmospheric pressure for not very excited plasmas.

Higher electric fields (more than about $100 \mathrm{Td}$ for air as follows from Figure 1) lead to more efficient excitation of electronic degree of freedom, dissociation and ionization processes (iii) than vibrational excitation (ii). As with vibrational relaxation, the electron relaxation rate $(\mathrm{ET})$ depends even more on the nature of the excited molecules and the composition of the plasma. However, unlike to 
VT relaxation, electron relaxation can be quite fast (see [3] and references therein).

A detailed model of the mechanism for rapid heating of nitrogen and air in gas discharges in relatively high electric fields was presented in [3]. According to this model, the gas is heated mainly during reactions of preliminary dissociation of highly excited electronic states of oxygen molecules which are produced either by electron impact or during the quenching of excited states of $\mathrm{N}_{2}$ molecules. The results of the model calculations were found to adequately describe available experimental data on the dynamics of air heating in gas-discharge plasmas.

It was shown in [3] that, over a broad range of reduced electric fields, the fraction of discharge power that goes into heating the gas is fixed; specifically, it is the fraction that is expended on the excitation of the electronic degrees of freedom of molecules ( $28 \%$ for discharges in air).

The present paper provides an analysis of the timeresolved dynamics of gas heating in pulsed discharges.

\section{The model}

A zero-dimensional adiabatic multi-temperature model is used to describe the dynamics of species and gas temperature changes. This state-specific model of $\mathrm{N}_{2}-\mathrm{O}_{2}$ mixture includes a set of about 430 chemical reactions with the participation of 44 states, namely, molecules $\mathrm{N}_{2}\left(\mathrm{X}^{1}, v=\right.$ $0-8), \mathrm{N}_{2}\left(\mathrm{~A}^{3}, \mathrm{~B}^{3}, \mathrm{a}^{1}, \mathrm{C}^{3}\right), \mathrm{O}_{2}\left(\mathrm{X}^{3}, v=0-4\right), \mathrm{O}_{2}\left(\mathrm{a}^{1}, \mathrm{~b}^{1}\right.$, $4.5 \mathrm{eV}), \mathrm{O}_{3}$, NO, atoms $\mathrm{N}\left({ }^{4} \mathrm{~S},{ }^{2} \mathrm{D},{ }^{2} \mathrm{P}\right), \mathrm{O}\left({ }^{3} \mathrm{P},{ }^{1} \mathrm{D},{ }^{1} \mathrm{~S}\right)$, positive ions $\mathrm{N}^{+}, \mathrm{N}_{2}^{+}, \mathrm{N}_{3}^{+}, \mathrm{N}_{4}^{+}, \mathrm{O}^{+}, \mathrm{O}_{2}^{+}, \mathrm{O}_{4}^{+}, \mathrm{NO}^{+}, \mathrm{O}_{2}^{+} \mathrm{N}_{2}$, negative ions $\mathrm{O}^{-}, \mathrm{O}_{2}^{-}, \mathrm{O}_{3}^{-}, \mathrm{O}_{4}^{-}, \mathrm{NO}^{-}$and electrons $\mathrm{E}$.
The model also accounts the excitation of other electronic states of nitrogen and oxygen, but assumes instantaneous relaxation $\mathrm{N}_{2}\left(\mathrm{~W}^{3}, \mathrm{~B}^{\prime 3}\right) \rightarrow \mathrm{N}_{2}\left(\mathrm{~B}^{3}\right), \mathrm{N}_{2}\left(\mathrm{a}^{1}, \mathrm{w}^{1}\right) \rightarrow$ $\mathrm{N}_{2}\left(\mathrm{a}^{\prime 1}\right)$ and $\mathrm{N}_{2}\left(\mathrm{E}^{3}, \mathrm{a}{ }^{1}\right) \rightarrow \mathrm{N}_{2}\left(\mathrm{C}^{3}\right)$. The generalized level $\mathrm{O}_{2}(4.5 \mathrm{eV})$ corresponds to $\mathrm{O}_{2}\left(\mathrm{~A}^{3}, \mathrm{C}^{3}\right)$ and $\mathrm{O}_{2}\left(\mathrm{c}^{1}\right)$ states. These assumptions will bring a little artificial heating in the model due to the slightly different energies of species.

The dynamics of species density $\left[N_{i}\right]$ is described by kinetic equation

$$
\frac{d\left[N_{i}\right]}{d t}=S_{i}=\sum_{j} S_{i j}
$$

where the source term $S_{i}$ for species $i$ consists of a sum of corresponding reaction sources $S_{i j}$. For example, this source term for reaction

$$
a N_{i}+b N_{l}+\ldots \rightarrow a^{\prime} N_{i}+c N_{m}+\ldots
$$

can be expressed using reaction rate $R_{j}$ and reaction constant rate $k_{j}$ as follows

$$
\begin{aligned}
R_{j} & =k_{j}\left[N_{i}\right]^{a}\left[N_{l}\right]^{b} \ldots \\
S_{i j} & =\left(a^{\prime}-a\right) R_{j}
\end{aligned}
$$

The list of species and reactions are converted automatically to the system of kinetic equations and solved numerically using ZDPlasKin tool [4]. Transport parameters and constant rates for electron-neutral interactions are calculated using build-in into the package BOLSIG+ solver. The density of 20 species, namely $\mathrm{N}_{2}\left(\mathrm{X}^{1}, v=0-8\right)$, $\mathrm{O}_{2}\left(\mathrm{X}^{3}, v=0-4\right), \mathrm{N}_{2}\left(\mathrm{~A}^{3}\right), \mathrm{O}_{2}\left(\mathrm{a}^{1}\right), \mathrm{N}, \mathrm{O}, \mathrm{NO}, \mathrm{O}_{3}$ and 78 collisional processes including super-elastic collisions with 
above-mentioned states are used solving the Boltzmann equation for electron energy distribution function. The cross-section database for electron-neutral collisions distributed with ZDPlasKin package has been extended with the cross sections available in [5].

The reaction constant rates between radicals, electronically- So, the moment of significant gas heating due to VT excited species, atoms and ions are mainly taken from [2]. The rates of some relaxation processes of excited atoms are taken from $[6-8]$

\subsection{Vibrational excitation}

The population of vibrational levels of molecules (both excitation and depopulation) is controlled by electron impact and collisions with heavy species.

The constant rates of $\mathrm{VT}$ relaxation $k_{10}$ of $\mathrm{N}_{2}\left(\mathrm{X}^{1}, v=\right.$ 1) and $\mathrm{O}_{2}\left(\mathrm{X}^{3}, v=1\right)$ molecules on $\mathrm{N}_{2}, \mathrm{O}_{2}, \mathrm{~N}$ and $\mathrm{O}$ species were taken from [2] and were applied for all vibrational levels using the harmonic oscillator model $k_{v+1, v}=$ $(v+1) k_{10}$. A set of reverse processes was built with constant rates $k_{v, v+1}$ obtained using the principle of detailed balance.

Such a vibrational model is quite simple, it uses a rough dependence of rate change with vibrational number and does not account multiquantum V-T transitions in atom-diatom collisions at all that is highly important for vibrational distribution; however the present work does not focus on accurate computation of vibrational population and readers are directed to the numerous publications on this subject (see, for example, [7-10] and references therein).
Fast gas heating due to plasmachemical transformation is usually the dominant heating process until the moment when VT relaxation comes into play. This is true for quite a wide range of electric fields apart from very high fields.

relaxation is used here as the time limit when gas heating occurs mainly due to relaxation of electronically excited species. More realistic vibrational model must be involved for correct description of gas heating after this time limit (like it was done in [8], for instance).

\subsection{The temperatures of species}

In the model, it is assumed that the temperature of neutral heavy species (i.e. the gas temperature $T_{\text {gas }}$ ) is constant in time and equal to room temperature in all results presented in this paper. This is done to simplify the analysis of the gas heating dynamic, and only the heating rate is calculated. The details of this procedure will be presented later in the text.

As mentioned earlier, the electron effective temperature (equal to $2 / 3$ of mean electron energy) is obtained using BOLSIG+ solver.

Like electrons, ions accelerate in electric fields and their temperature may differ significantly from the temperature of neutral species. Mean ion velocity $\left\langle v_{i}\right\rangle$ can be expressed through the mobility $\mu$ and the local electric field $E$ as $\left\langle v_{i}\right\rangle=\mu E$. The $\mu N$ values of the mobility times gas density $N$ measured in [11] are 8.0, 4.1, 6.1, and $7.0 \times 10^{19}(\mathrm{~V} \mathrm{~cm} \mathrm{~s})^{-1}$ for $\mathrm{N}^{+}, \mathrm{N}_{2}^{+}, \mathrm{N}_{3}^{+}$and $\mathrm{N}_{4}^{+}$ions, respec- 
tively, moving in nitrogen. These values are also adopted for the corresponding oxygen ions, both positive and negative.

The ion temperature $T_{i}$ can be expressed according to $[12]$ as

$$
T_{i}=T_{g a s}+\Delta T_{i}, \quad \Delta T_{i}=\frac{2}{3 k} m_{i}\left\langle v_{i}\right\rangle^{2}
$$

The effective temperature $T_{\text {eff }}$ corresponding to ionneutral collisions in the system of center of mass is given then by

$$
T_{e f f}=T_{g a s}+\Delta T_{e f f}, \quad \Delta T_{e f f}=\frac{2}{3 k} \frac{m_{i} m_{n}}{m_{i}+m_{n}}\left\langle v_{i}\right\rangle^{2}
$$

Here, $k$ is the Boltzmann constant, $m_{i}$ and $m_{n}$ are the mass of ions and of neutral species, respectively.

\subsection{Typical plasma composition in dry air}

The nitrogen-oxygen thermal equilibrium distribution of species in 4:1 proportion with $T_{\text {gas }}$ temperature at atmospheric pressure is used as the initial conditions in simulations. Verification was carried out that this distribution satisfies time-independent (stationary) conditions for the application of the constructed kinetic scheme.

The changes of selected species at a constant reduced field of $E / N=100 \mathrm{Td}$ is presented in Figures 2 and 3 for a fixed electron density of $\left[N_{e}\right]=1 \mathrm{~cm}^{-3}$ and $\left[N_{e}\right]=10^{12}$ $\mathrm{cm}^{-3}$, respectively. Here and below we will fix the electron density at different levels to cover a wide range of plasma densities. This is required in the present paper due to the using 0D approach when we do not simulate transport of species.

Three time periods may be selected in the changes of species. The first one is characterized by a relatively low excitation degree of gas and almost exponential growth of species which are produced in electron collisions with molecules in the ground state. Significant changes in species distribution take place in the following time interval when processes with atomic species begin to play an important role in plasma composition. The last time interval is characterized by a significant accumulation of long-life species like $\mathrm{O}_{3}$ and $\mathrm{NO}$.

Depending on conditions, the composition of plasma can reach a stationary solution when the processes of population and depopulation of each state compensate each other as presented, for instance, in Figure 3.

\subsection{Gas heating}

These are electrons that are usually responsible for energy transmission from an external power source to gas. The difference of this deposited energy and losses due to plasma expansion, thermal conductivity and radiative losses, normally, goes on excitation of internal degrees of freedom of gas and increases the temperatures of species.

The heating rate in the adiabatic case can be expressed using the energy balance equation. The electric power deposited to gas $P_{\text {ext }}$ redistributes between the translational degree of freedom of electrons $P_{\text {elec }}$ and gas $P_{\text {gas }}$ and internal degrees of freedom of gas $P_{\text {chem }}$ : 


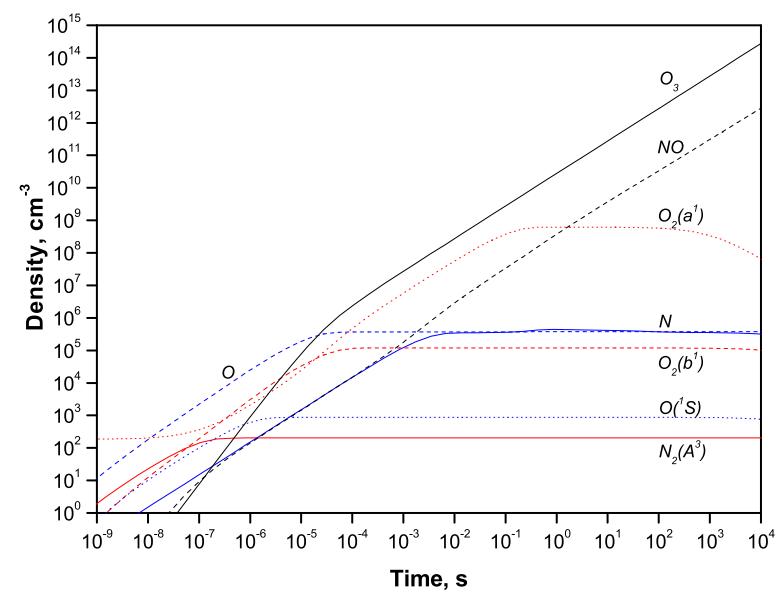

Fig. 2. Density of selected species in dry air at atmospheric pressure and constant room temperature under the action of a constant electric field of $100 \mathrm{Td}$ and assuming a constant density of electrons of $1 \mathrm{~cm}^{-3}$.

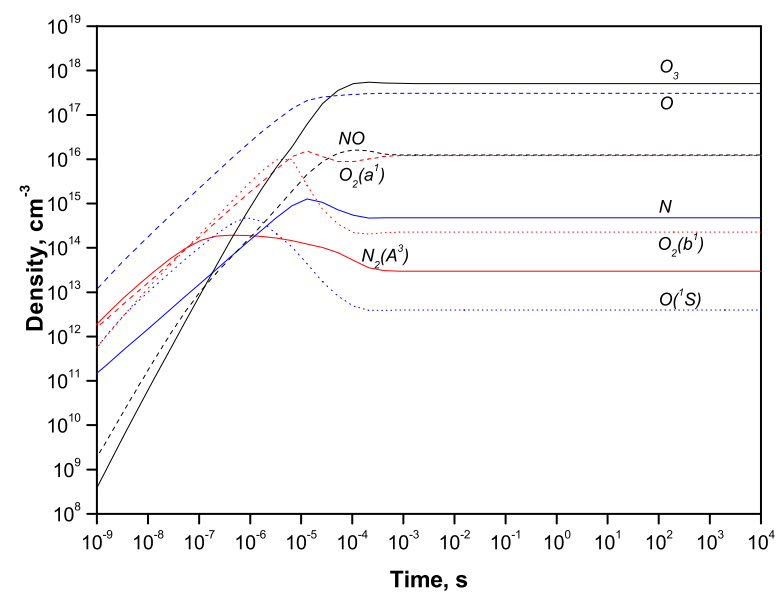

Fig. 3. Density of selected species in dry air at atmospheric pressure and constant room temperature under the action of a constant electric field of $100 \mathrm{Td}$ and assuming a constant density of electrons of $10^{12} \mathrm{~cm}^{-3}$.

$$
P_{\text {ext }}=P_{\text {elec }}+P_{\text {gas }}+P_{\text {chem }}
$$

These terms can be expressed as

$$
\begin{aligned}
P_{\text {ext }} & =e\left[N_{e}\right] v_{e} E \\
P_{\text {elec }} & =\frac{3}{2} \frac{d\left(\left[N_{e}\right] T_{e}\right)}{d t} \\
P_{\text {gas }} & =\frac{1}{\gamma-1} \frac{d\left(N T_{\text {gas }}\right)}{d t} \\
P_{\text {chem }} & =\sum_{i} Q_{i} \frac{d\left[N_{i}\right]}{d t}
\end{aligned}
$$

where $e$ is the elementary charge, $\left[N_{e}\right]$ is the electron density, $v_{e}$ is the drift velocity of electrons, $E$ is the reduced field, $T_{e}$ is the effective electron temperature, $N=\sum_{i}\left[N_{i}\right]$ is the total gas density, $\gamma=1.4$ is the specific gas heat ratio for vibrationally non-excited air and $Q_{i}$ is the potential energy of species $i$.

We note that $P_{\text {chem }}$ can be positive or negative depending on the excitation/quenching rates of the species, i.e. the sign of $d\left[N_{i}\right] / d t$ terms. In the case of negative $P_{\text {chem }}$, this energy is liberated and goes to excitation of other degrees of freedom including heating the gas.

The combination of primary equation (7) with equations (8-11) allows gas temperature calculation using an ordinary differential equation that is sufficient for practical purposes. However, we will split the energies to highlight the main channels of fast gas heating.

Electric power $P_{\text {ext }}$ deposited into gas in collisions of electrons with heavy species goes to direct gas heating $P_{e x t}^{T, R}$, vibrational excitation $P_{e x t}^{V}$ and excitation of electronic levels $P_{\text {ext }}^{E}$. Here and below we refer to the electronic excitation which, in fact, includes not only processes of 
direct excitation of electronic levels but also dissociation, ionization, attachment and recombination processes, i.e. excitation of all internal degrees of freedom of gas except rotational and vibrational systems. By doing so, the total electric power can be expressed as

$$
P_{e x t}=P_{e x t}^{T, R}+P_{e x t}^{V}+P_{e x t}^{E}
$$

These partial excitation powers can be expressed using the rate $R_{j}(3)$ and the threshold $\varepsilon_{j}$ of corresponding reactions:

$$
P_{\text {ext }}=\sum_{j} \varepsilon_{j} R_{j}
$$

where the sum is taken over all processes of electron vibrational excitation $\left(P_{e x t}^{V}\right)$ or over all processes of excitation of electronic levels in electron collisions $\left(P_{\text {ext }}^{E}\right)$, respectively. We note that super-elastic collisions are also included in the latter expression (13) with negative threshold energies.

In much the same way, $P_{\text {chem }}$ power consists of vibrational and electronic subsystems $P_{c h e m}^{V}$ and $P_{\text {chem }}^{E}$ :

$$
\begin{aligned}
& P_{\text {chem }}=P_{\text {chem }}^{V}+P_{\text {chem }}^{E} \\
& P_{\text {chem }}^{V}=\sum_{i} Q_{i} \frac{d\left[N_{i}\right]}{d t}, \text { over all vibrational states } \\
& P_{\text {chem }}^{E}=\sum_{i} Q_{i} \frac{d\left[N_{i}\right]}{d t}, \text { over all electronic states }
\end{aligned}
$$

Primary equation (7) can now be expanded using equations (12) and (14) as

$$
P_{g a s}=P_{e x t}^{T, R}-P_{e l e c}+\left(P_{e x t}^{V}-P_{c h e m}^{V}\right)+\left(P_{e x t}^{E}-P_{c h e m}^{E}\right)
$$

Thus, the difference between power deposited to vibrations (electronic states) $P_{e x t}^{V(E)}$ and energy stored per unit time into vibrations (electronic states) $P_{c h e m}^{V(E)}$ is attributed to gas heating.

To make quantitative analysis, the heating rate $\eta_{\Sigma}$ is defined using (12) as follows

$$
\eta_{\Sigma}=\frac{P_{g a s}}{P_{\text {ext }}}=\frac{P_{\text {ext }}-\left(P_{\text {elec }}+P_{\text {chem }}\right)}{P_{\text {ext }}},
$$

this is, in fact, the instantaneous fraction of energy going to gas heating and the total electric energy being deposited into plasma.

To help understand the main mechanisms of gas heating, two other heating rate parameters are defined as

$$
\eta_{V}=\frac{P_{e x t}^{V}-P_{c h e m}^{V}}{P_{e x t}^{V}}, \text { and } \eta_{E}=\frac{P_{e x t}^{E}-P_{c h e m}^{E}}{P_{e x t}^{E}}
$$

both of which, in fact, show the fractions of power deposited into internal degrees of freedom of gas - vibrational and electronic, respectively - that heat the gas [3].

It should be noted here that relaxation and conversion of excited species are often accompanied by vibrational excitation of products, so a part of the difference $P_{e x t}^{E}-$ $P_{\text {chem }}^{E}$ can be redistributed into $P_{\text {chem }}^{V}$. For this reason, the present model over-estimates the rate of gas heating and can be used as the maximum possible heating rate in a pulsed discharge. We will estimate the accuracy of our model by comparing the results with the results of the detailed model [3]. 


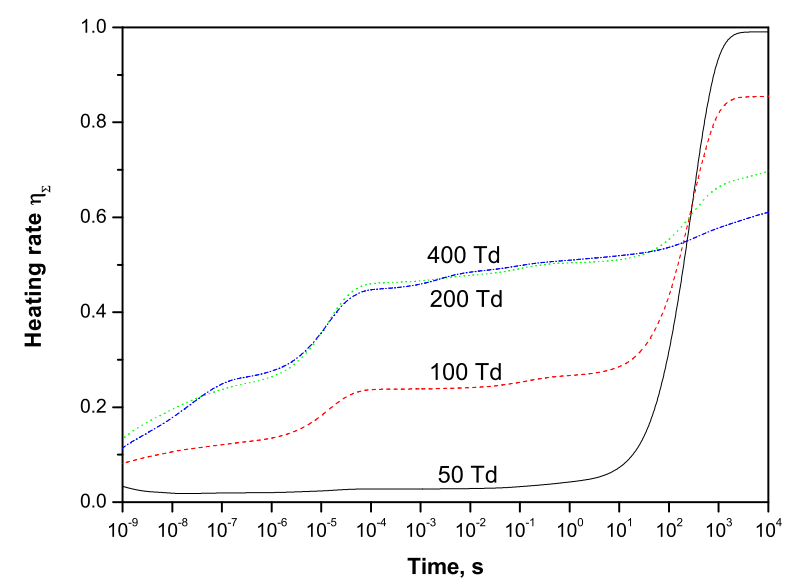

Fig. 4. Heating rate $\eta_{\Sigma}$ calculated for different electric fields in dry air at normal conditions assuming a constant density of electrons of $1 \mathrm{~cm}^{-3}$.

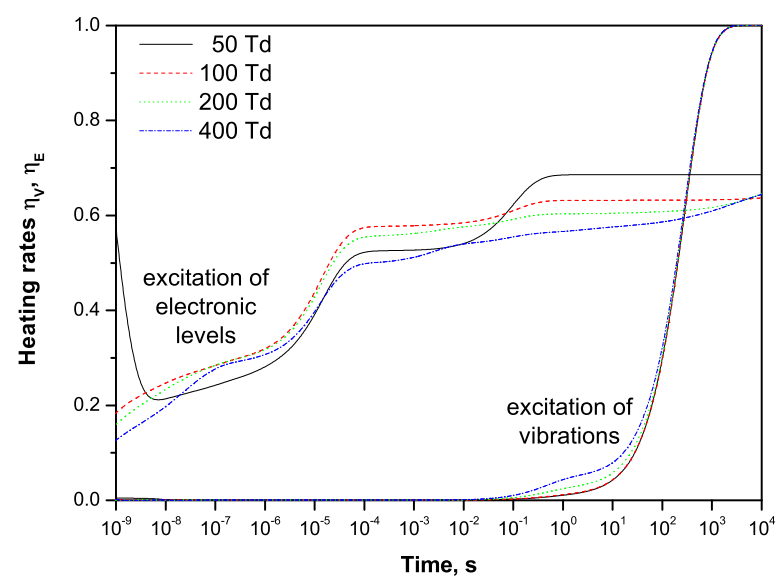

Fig. 5. Heating rates $\eta_{V}$ and $\eta_{E}$ calculated for different electric fields in dry air at normal conditions assuming a constant density of electrons of $1 \mathrm{~cm}^{-3}$.

\subsection{Dynamics of fast gas heating}

All heating rates defined in the previous section are presented in Figures 4 and 5 for different electric fields in dry air at atmospheric pressure and room temperature assuming $1 \mathrm{~cm}^{-3}$ constant density of electrons.
The general tendency according to Figure 4 is that a higher field exhibits a higher fraction of total energy quickly going to gas heating. This rate not only depends on the value of the field but it changes in time due to changes in plasma composition. As mentioned early, a sharp increase in the heating rate in the Figure is associated with VT relaxation and can be used as the limit of time interval where gas heating occurs mainly due to fast relaxation of electronic degree of freedom of gas.

However, as follows from Figure 5, the gas heating rates due to excitation of vibrational and electronic degrees of freedom is almost independent of the value of applied field and changes in time due to changes of plasma composition.

This result was predicted in [3] using the detailed statespecific model in quasi-stationary conditions. The author of that paper has shown that about $28 \%$ of power spent on excitation of electronic degrees of freedom of molecules in similar conditions goes into heating the gas.

In the present simulations, the value $\eta_{E}=28 \%$ corresponds to time interval $\tau \simeq 10^{-7}-10^{-5} \mathrm{~s}$ only and increases to $\eta_{E}=50-70 \%$ at later time moments. We remained that atmospheric pressure is used here, so the characteristic times must be scaled with pressure (or, rather, with gas density $N)$ as $\tau \times N=$ const. It must be emphasized that we did not detect a significant pressure dependence of $\eta$ parameters, which is in agreement with results $[3]$. 


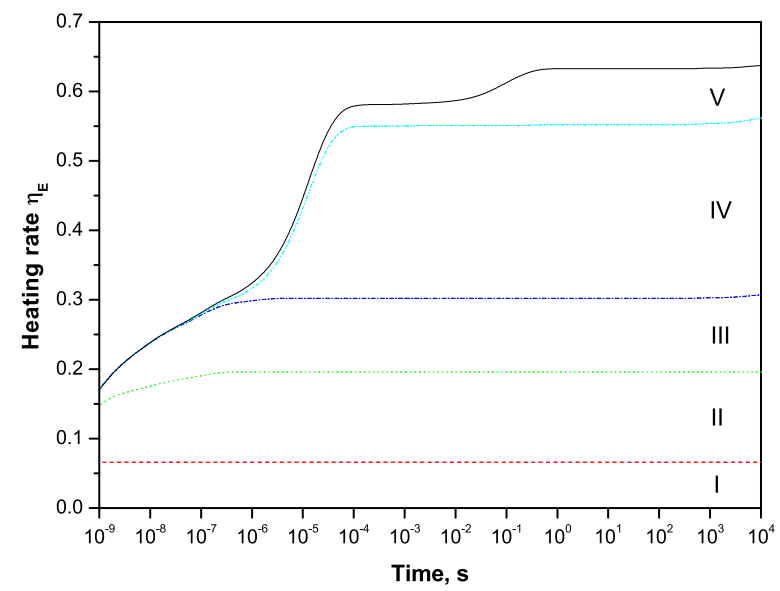

Fig. 6. Contribution of various reactions into heating rate $\eta_{E}$ calculated for a fixed electric field of $100 \mathrm{Td}$ in dry air at atmospheric pressure and room temperature assuming a constant density of electrons of $1 \mathrm{~cm}^{-3}$. See text for description.

The shape of the curves in Figures 4 and 5 leads us to suppose that the main heating channel changes in time, which is demonstrated in the following Figure.

Five main channels starting to work at different times are selected and presented in Figure 6. All of them are related to the temporal profile of the species participating in the corresponding process:

(I) Permanent in time contribution of about $6.6 \%$ is due to energy conversion in excitation of species by electron collisions. A part of this energy corresponds to the difference in threshold of the excitation processes and energy stored in products of the excitation. Another part comes from the assumption about the fast relaxation of some electronic states which was mentioned in the model description.
(II) Quenching of $\mathrm{N}_{2}\left(\mathrm{~A}^{3}, \mathrm{~B}^{3}, \mathrm{C}^{3}, \mathrm{a}^{\prime 1}\right)$ excited states in collisions with $\mathrm{O}_{2}$ (mainly) and $\mathrm{N}_{2}$ molecules. This contribution increases initially and then becomes a constant value as soon as the densities of involved species reach their stationary values.

(III) Quenching of $\mathrm{O}\left({ }^{1} \mathrm{D},{ }^{1} \mathrm{~S}\right)$ excited atoms by $\mathrm{O}_{2}, \mathrm{~N}_{2}$ and $\mathrm{O}_{3}$ molecules.

(IV) Three-body ozone $\mathrm{O}_{3}$ production in collisions with $\mathrm{N}_{2}$ and $\mathrm{O}_{2}$.

(V) Quenching of $\mathrm{O}_{2}\left(\mathrm{a}^{1}, \mathrm{~b}^{1}, 4.5 \mathrm{eV}\right)$ states by $\mathrm{O}_{2}, \mathrm{~N}_{2}$, $\mathrm{NO}$ and $\mathrm{O}_{3}$ molecules.

This list of channels provides the means to predict the influence of the presence of various species in plasma on the gas heating rate.

\section{Strong gas excitation}

The low electron density case used in the previous section corresponds to the low-rate excitation of gas. However, to be expected that an increase in electron density could change the dynamics of gas heating. To check this influence, the results obtained with a variation of electron density are presented below.

The heating rate presented in Figures 7 and 8 is obtained in dry air at atmospheric pressure and room temperature for electric field of 100 and $400 \mathrm{Td}$ and electron densities of $1,10^{8}$ and $10^{12} \mathrm{~cm}^{-3}$.

We found that in spite of a significant acceleration the heating rate saturation point, i.e. the moment when $\eta$ gets very close to $100 \%$, the beginnings of corresponding cures are almost identical for a given electric field. We conclude 


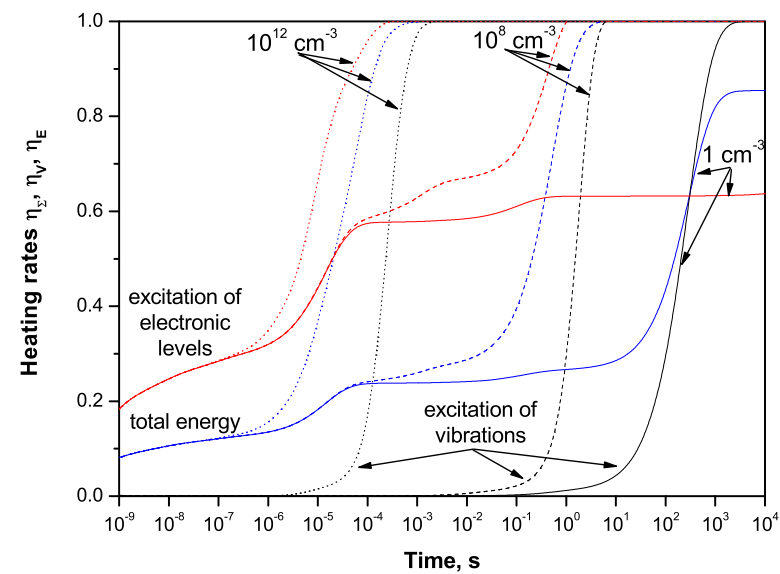

Fig. 7. Heating rates calculated for a fixed electric field of 100 Td in dry air at atmospheric pressure and room temperature with various densities of electrons.

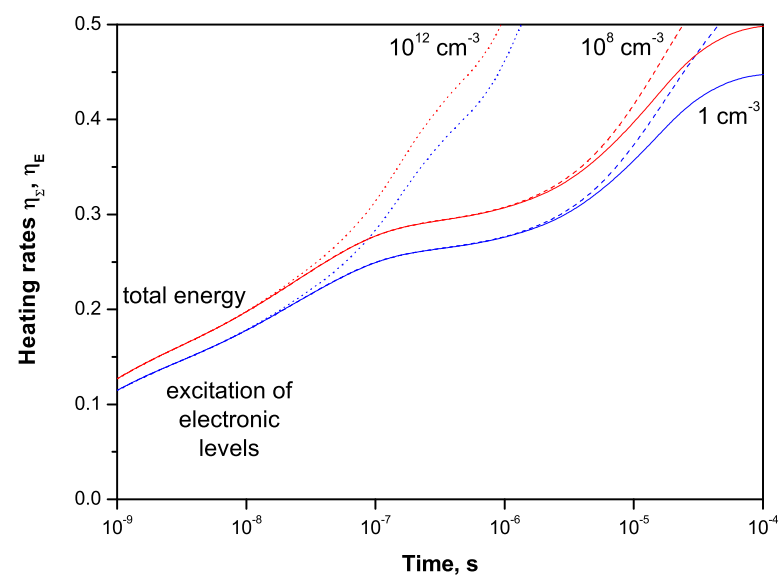

Fig. 8. Heating rates calculated for a fixed electric field of 400 $\mathrm{Td}$ in dry air at atmospheric pressure and room temperature with various densities of electrons.

here that an increase in the electron density or electric field accelerates the production of species and speeds up the gas heating does not affect this rate at the beginning.

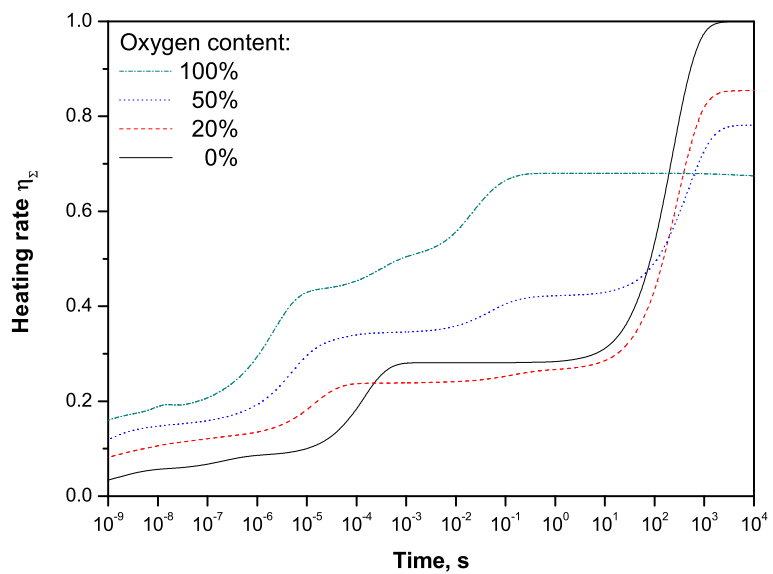

Fig. 9. Heating rate $\eta_{\Sigma}$ calculated for various content of oxygen in $\mathrm{N}_{2}-\mathrm{O}_{2}$ mixture at atmospheric pressure and room temperature. Constant electric field of $100 \mathrm{Td}$ and density of electrons of $1 \mathrm{~cm}^{-3}$ are assumed here.

\section{Variation of oxygen partial pressure}

A change of the oxygen content in plasma affects the heating rate presented in Figure 9.

This result was obtained in [3] where it was shown that lower oxygen content in the mixture leads to lower gas heating rate.

The main channels of gas heating in the case of pure oxygen differ slightly on those in a nitrogen-oxygen mixture and are presented in Figure 10:

(I) Permanent in time contribution of about $3.5 \%$ is due to energy conversion in excitation of species by electron collisions.

(II) Quenching of $\mathrm{O}\left({ }^{1} \mathrm{D}\right)$ excited atoms in collisions with $\mathrm{O}_{2}$.

(III) Quenching of $\mathrm{O}_{2}(4.5 \mathrm{eV})$ state by $\mathrm{O}_{2}$ molecules. 


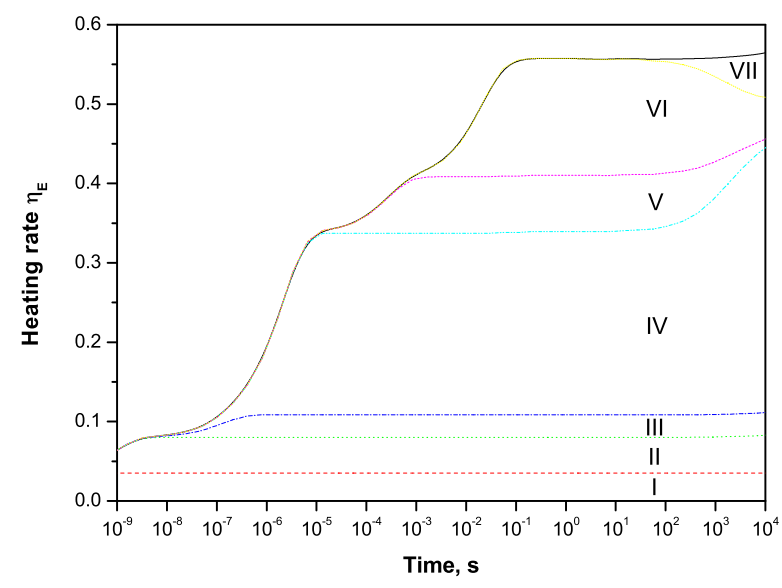

Fig. 10. Contribution of various reactions into heating rate $\eta_{E}$ calculated for a fixed electric field of $100 \mathrm{Td}$ for pure oxygen at atmospheric pressure and room temperature. Constant density of electrons of $1 \mathrm{~cm}^{-3}$ is assumed here. See text for description.

(IV) Three-body ozone $\mathrm{O}_{3}$ production in collisions with $\mathrm{O}_{2}$.

$(\mathrm{V}, \mathrm{VI})$ Quenching of $\mathrm{O}_{2}\left(\mathrm{~b}^{1}\right)$ and $\mathrm{O}_{2}\left(\mathrm{a}^{1}\right)$ states by $\mathrm{O}_{2}$ molecules, respectively.

(VII) Quenching of $\mathrm{O}_{2}\left(\mathrm{~b}^{1}\right)$ state by $\mathrm{O}_{3}$ molecules.

\section{Conclusions}

The dynamics of gas heating in a fast pulsed discharge in $\mathrm{N}_{2}-\mathrm{O}_{2}$ mixture is analyzed in the present work. Using a state-specific plasmachemical model and energy conservation equation, the heating rate, i.e. the instantaneous fraction of energy going into gas heating and the total electric energy depositing into different degrees of freedom of gas, is calculated.

It is shown that the heating rate changes in time according to the changes of species involved in the heating processes and exhibits an increasing dependence in time. The results obtained show that higher electron density, electric field or partial pressure of oxygen leads to faster growth of this dependence. However, the gas heating rates due to excitation of vibrational and electronic degrees of freedom is almost independent of the value of the applied field, at least in the checked range of 50-400 Td. This is in agreement with results [3].

We note that in neglecting vibrational excitation of products in quenching processes of electronic states, the developed model over-estimates the rate of gas heating and gives the maximum heating rate in pulsed discharges. This overestimation is probably about double based on a comparison of results obtained in the present work with those of the detailed model [3] but this requires additional checking.

The developed model can therefore be used in the simulation of plasmas produced by pulsed discharges at elevated electric fields.

The authors are grateful to Dr. Nikolay Popov from the ScientificResearch Institute of Nuclear Physics of Moscow State University for his criticisms and valuable comments.

\section{References}

1. Hagelaar G.J.M. and Pitchford L.C., Solving the Boltzmann equation to obtain electron transport coefficients and rate coefficients for fluid models, Plasma Sources Sci. Technol. 14 722-733 (2005). 
2. Capitelli M., Ferreira C.M., Gordiets B.F., Osipov A.I., Plasma Kinetics in Atmospheric Gases, Springer, Berlin, 2000.

3. Popov N., Investigation of the mechanisms for rapid heating of nitrogen and air in gas discharges, Plasma Physics Reports 27 886-896 (2001).

4. Pancheshnyi S., Eismann B., Hagelaar G. and Pitchford L. Computer code ZDPlasKin, University of Toulouse, LAPLACE, CNRS-UPS-INP, Toulouse, France, 2008, http://www.zdplaskin.univ-tlse.fr.

5. Kinema Research \& Software, Humid air chemistry database, http://www.kinema.com/download.htm.

6. Kossyi I A, Kostinsky A Yu, Matveyev A A and Silakov $\mathrm{V} \mathrm{P}$, Kinetic scheme on the non-equilibrium discharge in nitrogen-oxygen mixtures, Plasma Sources Sci. Technol. 1 207 (1992).

7. Gordiets B.F., Ferreira C.M., Guerra V.L., Loureiro J.M.A.H., Nahorny J., Pagnon D., Touzeau M. and Vialle M., Kinetic model of a low-pressure $\mathrm{N}_{2}-\mathrm{O}_{2}$ flowing glow discharge, IEEE Transactions on Plasma Science 23 750-768 (1995).

8. Guerra V., Sa P.A. and Loureiro J., Kinetic modeling of low-pressure nitrogen discharges and post-discharges, Eur. Phys. J. Appl. Phys. 28 125-152 (2004).

9. Esposito F. and Capitelli M., QCT calculations for the process $N_{2}(v)+N \rightarrow N_{2}\left(v^{\prime}\right)+N$ in the whole vibrational range, Chemical Physics Letters 418 581(2006).

10. Esposito F., Armenise I., Capitta G. and Capitelli M., $\mathrm{O}-\mathrm{O}_{2}$ state-to-state vibrational relaxation and dissociation rates based on quasiclassical calculations, Chemical Physics 351 91(2008).
11. McKnight L.G., McAfee K.B. and Sipler D.P., Low-field drift velocities and reactions of nitrogen ions in nitrogen, Physical Review 164 62-70 (1967).

12. Wannier G.H., Motion of gaseous ions in strong electric fields, Bell Syst. Tech. 32170 (1953). 